\title{
The Transverse Momentum Dependence of Quark Fragmentation Functions from Cascade Models
}

\author{
E.H. de Groot and J. Engels \\ Department of Theoretical Physics, University of Bielefeld, D-4800 Bielefeld, Federal Republic of Germany
}

\begin{abstract}
A covariant generalization of the onedimensional cascade model for quark fragmentation functions is presented, so as to include the transverse momentum behaviour and the possibility to produce different particles at different vertices along the chain. In the scaling limit the exact solution is given, if the primordial function is of the type $\alpha z^{\alpha-1} \cdot T\left(p_{T}\right)$. For the more general case of factorizing primordial functions an analytic expression for the seagull effect is derived, which turns out to be independent of the function $T\left(p_{T}\right)$.
\end{abstract}

\section{Introduction}

The existence of jets of hadrons in $e^{+} e^{-}$reactions gets more and more convincing experimental support [1].

Preliminary measurements in the energy region of 8-9.4 GeV at DESY indeed show that many of the events have a clear two-jet structure [2]. It is commonly believed that these jets are of the same type as those observed in other high energy processes initiated either by leptons or hadrons and furthermore that these jets are produced by the decay or breakup of quarks.

The emission of mesons from the quark is assumed to proceed stepwise in fundamental subprocesses of the type quark $\rightarrow$ meson + quark. Such cascade models are usually described in terms of integral equations in the variable $z$, the momentum fraction of the initial quark, for the multiplicity, the quark fragmentation functions $D_{q}^{h}(z)$, double decay distributions etc. Explicit solutions of these equations for primordial functions (input vertex functions for the fundamental subprocess) of the power type or constant + power type have been given by the authors of [3-6]. They also nicely demonstrate the development of a platéau connected with the $1 / z$ dependence near $z=0$ of the fragmentation functions for all vertex functions. Other problems such as the various conservation laws or the inclusion of baryons in such models have also been studied in $[7-9]$.

However, the important question of the transverse momentum of particles produced in a quark jet has up to now been either ignored or it was treated in an unsatisfactory way. Field and Feynman [5] allowed in their Monte Carlo calculation for a transverse momentum of the quark decay products, but this was not done in the framework of a three dimensional cascade model ${ }^{1}$, with the consequence that there was no seagull effect for the directly produced particles. Only the introduction of decaying resonances (in [5] vectormesons) led to a dependence on $z$ of the average transverse momentum of the pseudoscalar mesons.

The plan of our paper is as follows. Section 2 contains the details of a generalized covariant cascade model for quark fragmentation functions of mesons and any number of quark flavours. In section 3 we consider the scaling limit of the model and give the solution for primordial functions of the type $\alpha z^{\alpha-1} . T\left(p_{T}\right)$. For all factorizing primordial functions we derive an analytic expression for the seagull effect. Numerical examples of the transverse momentum implications for different models are calculated in section 4, which is followed by a summary and conclusions.

\section{The Generalized Cascade Model}

In the treatment of $N$ different quark flavours we shall follow partly the work of Sukhatme [6]. Consider the fundamental subprocess

$q_{i}(p) \rightarrow M_{i \bar{j}}(k)+q_{j}\left(p^{\prime}\right)$,

\footnotetext{
${ }^{1}$ Models of that type have been formulated in a somewhat different physical context in $[10,11]$.
} 
where the indices denote the flavours, $p$ and $p^{\prime}$ the quark four momenta, $k$ the meson four momentum and $M_{i \bar{j}}$ is the meson built from $q_{i} \bar{q}_{j}$. Of course we have

$p=p^{\prime}+k$.

Neglecting the spins, the $\left(i j M_{i j}\right)$-vertex is described fully by the so called primordial function $F_{i j}\left(p, p^{\prime}\right)^{2}$. Let us assume for simplicity that

$F_{i j}\left(p, p^{\prime}\right)=P_{i j} F\left(p, p^{\prime}\right)$,

where $P_{i j}$ is the total probability of obtaining an $\left(i j M_{i j}\right)$-vertex in a breakup of a quark of flavour $i$. Therefore we must have

$\sum_{j=1}^{N} P_{i j}=1$.

In addition Sukhatme assumes that the $N \times N$ probability matrix $P$ is symmetric, which we think is reasonable, but it is not necessary for the following considerations. The primordial function is normalized such that

$\int d p^{\prime} p_{0}^{\prime} F\left(p, p^{\prime}\right)=p_{0}$,

where $d p^{\prime}=d^{3} p^{\prime} / p_{0}^{\prime}$. From this normalization it is clear that the invariant probability density is $p_{0}^{\prime} F / p_{0}$. Working with $F$ simplifies the following equations for the meson distributions. Let $G_{i j}^{(n)}\left(p, p^{\prime}\right)$ denote the inclusive distribution for a quark $q_{j}$ of four momentum $p^{\prime}$ which originates from the $n^{\prime}$ th vertex of a cascade which started with a quark $q_{i}$ of four momentum $p$, then

$G_{i j}^{(n)}\left(p, p^{\prime}\right)=\int d p_{1} \ldots d p_{n-1} \sum_{K_{1}, \ldots, K_{n-1}} F_{i K_{1}}\left(p, p_{1}\right)$

$\cdot F_{K_{1} K_{2}}\left(p_{1}, p_{2}\right) \ldots F_{K_{n-1} j}\left(p_{n-1}, p\right)$

$=\left(P^{n}\right)_{i j} \int d p_{1} \ldots d p_{n-1} F\left(p, p_{1}\right) \ldots F\left(p_{n-1}, p^{\prime}\right)$

and $G_{i j}^{(n)}\left(p, p^{\prime}\right)$ can be written as

$G_{i j}^{(n)}\left(p, p^{\prime}\right)=\left(P^{n}\right)_{i j} G^{(n)}\left(p, p^{\prime}\right)$,

with

$G^{(0)}\left(p, p^{\prime}\right)=\delta\left(p-p^{\prime}\right)=p_{0} \delta^{(3)}\left(p-p^{\prime}\right)$,

$G^{(1)}\left(p, p^{\prime}\right)=F\left(p, p^{\prime}\right)$.

Obviously $G^{(n)}\left(p, p^{\prime}\right)$ obeys the recursive relations

$G^{(n)}\left(p, p^{\prime}\right)=\int \AA^{\prime \prime} G^{(n-1)}\left(p, p^{\prime \prime}\right) F\left(p^{\prime \prime}, p^{\prime}\right)$,

$G^{(n)}\left(p, p^{\prime}\right)=\int \bar{d} p^{\prime \prime} F\left(p, p^{\prime \prime}\right) G^{(n-1)}\left(p^{\prime \prime}, p^{\prime}\right)$.

The total inclusive quark distribution is given by

$G_{i j}\left(p, p^{\prime}\right)=\sum_{n=0}^{\infty}\left(P_{n}\right)_{i j} G^{(n)}\left(p, p^{\prime}\right)$.

As $p^{\prime} G / p_{0}$ is the invariant quantity due to Eq. (5)

\footnotetext{
2 Throughout the paper we shall denote the four momentum dependent functions with capital letters, the Fourier transformed and/or one dimensional quantities with small letters.
}

we have

$G_{i j}\left(p, p^{\prime}\right)=\frac{p_{0}}{\sigma} \frac{d^{3} \sigma}{d^{3} p^{\prime}}$

Suppose the probability matrix can be diagonalized, which is true for real symmetric matrices and also for the kind of matrix Field and Feynman [5] use, since their matrix is a projection operator, then we can write

$P=B P_{D} B^{-1}$,

where $P_{D}$ is the diagonal matrix, containing in its main diagonal the eigenvalues $\lambda_{i}$ of $P$.

As a consequence Eq. (13) can be written as

$G_{i j}\left(p, p^{\prime}\right)=\delta_{i j} \delta\left(p-p^{\prime}\right)+\sum_{m=1}^{N} B_{i m} B_{m j}^{-1} \lambda_{m} G^{\lambda_{m}}\left(p, p^{\prime}\right)$,

with the generating function

$G^{\lambda}\left(p, p^{\prime}\right)=\sum_{n=1}^{\infty} \lambda^{n-1} G^{(n)}\left(p, p^{\prime}\right)$,

which implies

$G^{(n)}\left(p, p^{\prime}\right)=\left.\frac{1}{(n-1) !} \frac{\partial^{n-1}}{\partial \lambda^{n-1}} G^{\lambda}\left(p, p^{\prime}\right)\right|_{\lambda=0}$.

Multiplying Eqs. (11) and (12) by $\lambda^{n-1}$ and summing leads to the equivalent equations

$G^{\lambda}\left(p, p^{\prime}\right)=F\left(p, p^{\prime}\right)+\lambda \int d p^{\prime \prime} G^{\lambda}\left(p, p^{\prime \prime}\right) F\left(p^{\prime \prime}, p^{\prime}\right)$,

$G^{\lambda}\left(p, p^{\prime}\right)=F\left(p, p^{\prime}\right)+\lambda \int d p^{\prime \prime} F\left(p, p^{\prime \prime}\right) G^{\lambda}\left(p^{\prime \prime}, p^{\prime}\right)$.

It is now easy to derive from the quark distribution at the $(n-1)$ th vertex the inclusive distribution $D_{i j ; l}^{(n)}(p, k)$ for mesons $M_{i \bar{j}}(k)$ coming from the $n^{\prime}$ th vertex of a chain, which was started by a quark $q_{l}(p)$

$D_{i j ; l}^{(n)}(p, k)=\int d p^{\prime} G_{l i}^{(n-1)}\left(p, p^{\prime}\right) F_{i j}\left(p^{\prime}, p^{\prime}-k\right)$,

or

$D_{i j, l}^{(n)}(p, k)=P_{i j}\left(P^{n-1}\right)_{l i} D^{(n)}(p, k)$,

with $D^{(n)}(p, k) \equiv \int \nexists p^{\prime} G^{(n-1)}\left(p, p^{\prime}\right) F\left(p^{\prime}, p^{\prime}-k\right)$.

In the same manner as we did in the case of the quark distributions we arrive now at the total inclusive meson distribution

$D_{i j, l}(p, k)=P_{i j} \sum_{m=1}^{N} B_{l m} B_{m i}^{-1} D^{\lambda_{m}}(p, k)=\frac{p_{0}}{\sigma} \frac{d^{3} \sigma}{d^{3} k}$,

where

$D^{\lambda}(p, k)=\sum_{n=1}^{\infty} \lambda^{n-1} D^{(n)}(p, k)$.

For this generating function one finds

$D^{\lambda}(p, k)=F(p, p-k)+\lambda \int d p^{\prime} G^{\lambda}\left(p, p^{\prime}\right) F\left(p^{\prime}, p^{\prime}-k\right)$.

There exists also an integral equation for $D^{\lambda}$

$D^{\lambda}(p, k)=F(p, p-k)+\lambda \int d p^{\prime} F\left(p, p^{\prime}\right) D^{\lambda}\left(p^{\prime}, k\right)$, 
however, because of the greater symmetry of Eqs. (19) and (20) $\left[G^{2}\right.$ is the resolvant kernel of Eqs. (19) and (26)] it is more convenient to solve these and use then Eq. (25) to calculate $D^{\lambda}$.

\section{The Scaling Limit}

In going over to the scaling limit we neglect all masses and consider the momenta transverse to some given direction (which we take at the end to be the direction of three momentum of the cascade initiating quark) to be small compared to the energies of the corresponding particles. We then assume that

$F\left(p, p^{\prime}\right) \equiv F\left(\frac{p_{0}^{\prime}}{p_{0}} ; \mathbf{p}_{T}^{\prime}-\mathbf{p}_{T} \frac{p_{0}^{\prime}}{p_{0}}\right) \Theta\left(p_{0}-p_{0}^{\prime}\right)$

With

$z=p_{0}^{\prime} / p_{0} ; d p^{\prime}=\frac{d z}{z} d^{2} p_{T}^{\prime}$

Eq. (5) becomes

$\int d z d^{2} p_{T} F\left(z ; \mathbf{p}_{T}\right)=1$.

In fact, the choice, Eq. (27), of the transverse momentum dependence of $F$ guarantees the conservation of transverse momentum as well:

$\int \frac{d z}{z} d^{2} p_{T}^{\prime} \mathbf{p}_{T}^{\prime} F\left(z ; \mathbf{p}_{T}^{\prime}-\mathbf{p}_{T} z\right)$

$=\int \frac{d z}{z} d^{2} p_{T}^{\prime \prime}\left(\mathbf{p}_{T}^{\prime \prime}+\mathbf{p}_{T} z\right) F\left(z ; \mathbf{p}_{T}^{\prime \prime}\right)$

$=\int d z d^{2} p_{T}^{\prime \prime} F\left(z ; \mathbf{p}_{T}^{\prime \prime}\right) \mathbf{p}_{T}=\mathbf{p}_{T}$,

where we have used Eq. (29) and $F\left(z ; \mathbf{p}_{T}\right)=F\left(z ;-\mathbf{p}_{T}\right)$, which any reasonable primordial function should fulfill. In the same manner longitudinal momentum is conserved up to some higher corrections in $p_{T}^{\prime \prime} / p_{0}^{\prime}$. The form, Eq. (27), of $F$ in the scaling limit means, that the transverse momentum is taken with respect to the preceding momentum $\mathbf{p}$ and not the original longitudinal direction (up to higher corrections in $p_{T} / p_{0}$ ). This can be seen in Figure 1. It is often presumed that introducing $\mathbf{p}_{T}$ in a cascade model would lead to a random walk variation in the final $\left\langle p_{T}^{2}\right\rangle$ of the mesons $(\sim\langle n\rangle)$. This is not true in our case, but would have been if we e.g. had chosen $\mathbf{p}_{T}^{\prime}-\mathbf{p}_{T}$ instead of $\mathbf{p}_{T}^{\prime}-z \mathbf{p}_{T}$ in Eq. (27). Another reason for choosing this variable is that the invariant

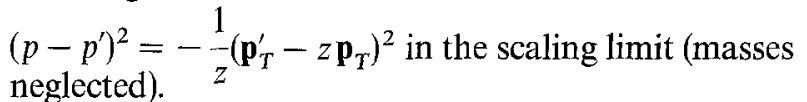
With Eq. (27) $G^{\lambda}$ and $D^{\lambda}$ turn out to be of the same form

$G^{\lambda}\left(p, p^{\prime}\right)=G^{\lambda}\left(\frac{p_{0}^{\prime}}{p_{0}} ; \mathbf{p}_{T}^{\prime}-\mathbf{p}_{T} \frac{p_{0}^{\prime}}{p_{0}}\right)$,
$D^{\lambda}\left(p, p^{\prime}\right)=D^{\lambda}\left(\frac{p_{0}^{\prime}}{p_{0}} ; \mathbf{p}_{T}^{\prime}-\mathbf{p}_{T} \frac{p_{0}^{\prime}}{p_{0}}\right)$.

Defining the Fourier transforms

$f(z, \boldsymbol{\rho})=\int d^{2} p_{T} e^{i \mathbf{p}_{T} \cdot \boldsymbol{\rho}} F\left(z ; \mathbf{p}_{T}\right)$,

$g^{\lambda}(z, \boldsymbol{\rho})=\int d^{2} p_{T} e^{i \mathbf{p}_{T} \cdot \rho} G^{\lambda}\left(z ; \mathbf{p}_{T}\right)$,

$d^{\lambda}(z, \boldsymbol{\rho})=\int d^{2} p_{T} e^{i \mathbf{p}_{T} \cdot \boldsymbol{\rho}} D^{\lambda}\left(z ; \mathbf{p}_{T}\right)$,

one obtains from eqs. (19), (20), (25) and (26)

$$
\begin{aligned}
& g^{\lambda}(z, \boldsymbol{\rho})=f(z, \boldsymbol{\rho})+\lambda \int_{z}^{1} \frac{d \xi}{\xi} g^{\lambda}\left(\xi, \frac{z}{\xi} \boldsymbol{\rho}\right) f\left(\frac{z}{\xi}, \boldsymbol{\rho}\right), \\
& g^{\lambda}(z, \boldsymbol{\rho})=f(z, \boldsymbol{\rho})+\lambda \int_{z}^{1} \frac{d \xi}{\xi} f\left(\xi, \frac{z}{\xi} \boldsymbol{\rho}\right) g^{\lambda}\left(\frac{z}{\xi}, \boldsymbol{\rho}\right), \\
& d^{\lambda}(z, \boldsymbol{\rho})=f(1-z,-\boldsymbol{\rho})+\lambda \int_{z}^{1} \frac{d \xi}{\xi} g^{\lambda}\left(\xi, \frac{z}{\xi} \boldsymbol{\rho}\right) \\
& f\left(1-\frac{z}{\xi},-\boldsymbol{\rho}\right), \\
& d^{\lambda}(z, \boldsymbol{\rho})=f(1-z,-\boldsymbol{\rho})+\lambda \int_{z}^{1} \frac{d \xi}{\xi} d^{\lambda}(\xi, \boldsymbol{\rho}) f\left(\frac{z}{\xi}, \xi \boldsymbol{\rho}\right) .
\end{aligned}
$$

For $\rho=0$ the last four equations reduce to those for the one dimensional case, since the point $\rho=0$ corresponds to integration over $\mathbf{p}_{T}$. Equation (37) for $\lambda=1$ is then the usually treated cascade equation. Of course, Eqs. (34) and (35) are equivalent and among eqs. (35), (36) and (37) only two are independent. The one dimensional equations are of course also obtained, when we assume that the primordial function $F\left(z ; \mathbf{p}_{T}\right)$ is proportional to $\delta\left(\mathbf{p}_{\tau}\right)$.

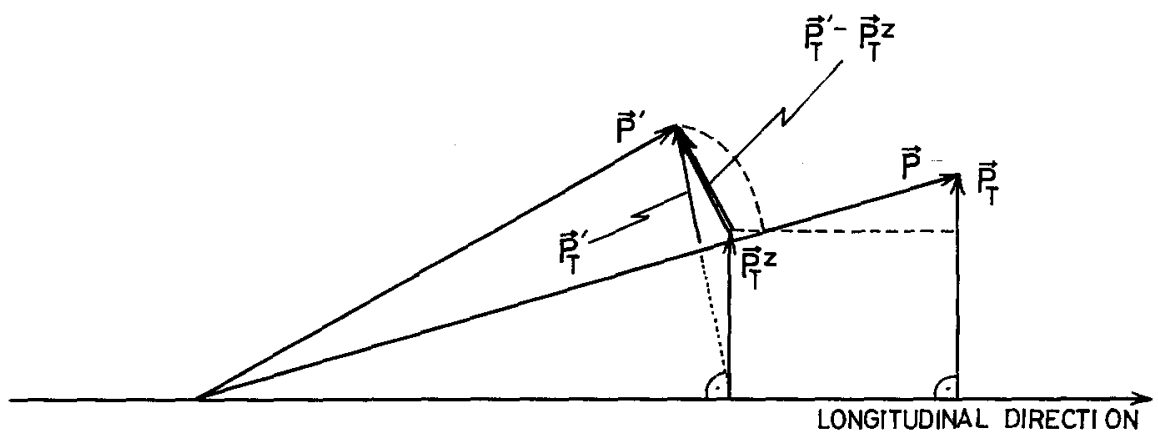

Fig. 1. Illustration of the argument of the scaling limit primordial function, Equation (27) 


\subsection{The One Dimensional Case}

For the following it is of interest to examine the one dimensional equations ${ }^{3}$

$g^{\lambda}(z)=f(z)+\lambda \int_{z}^{1} \frac{d \xi}{\xi} g^{\lambda}(\xi) f(z / \xi)$

$d^{\lambda}(z)=f(1-z)+\lambda \int_{z}^{1} \frac{d \xi}{\xi} g^{\lambda}(\xi) f\left(1-\frac{z}{\xi}\right)$,

$d^{\lambda}(z)=f(1-z)+\lambda \int_{z}^{1} \frac{d \xi}{\xi} d^{\lambda}(\xi) f(z / \xi)$

in more detail. Some of the results have been obtained for $\lambda=1$ already by other authors [3-6].

The general solution of Eq. (38) can most easily be obtained by taking either the Mellin transform of Eq. (38) or by defining

$x=-\ln z$,

$\bar{f}(x)=f(z)$,

$\bar{g}^{2}(x)=g^{2}(z)$

and Laplace transforming Eq. (38), resulting in the solution

$\bar{g}^{\lambda}(x)=L^{-1}\left(\frac{L(\bar{f})}{1-\lambda L(\bar{f})}\right)$,

where $L$ and $L^{-1}$ denote the Laplace and inverse Laplace transforms, respectively. Having found $g^{\lambda}$ in this way, $d^{\lambda}$ is immediately obtained by insertion of $g^{\lambda}$ into Eq. (39). That this method to calculate $d^{\lambda}$ is easier is clear from the fact, that if one tries to solve Eq. (40) via Laplace or Mellin transforms one also needs to know the transform of $f(1-z)$.

Defining the moments

$h_{\gamma}=\int_{0}^{1} d z z^{\gamma} h(z)$,

$h_{\gamma}^{-}=\int_{0}^{1} d z z^{\gamma} h(1-z)$,

one finds, multiplying (38) and (39) by $z^{\gamma}$ and integrating

$g_{\gamma}^{\lambda}=\frac{f_{\gamma}}{1-\lambda f_{\gamma}}$,

$d_{\gamma}^{\lambda}=\frac{f_{\gamma}^{-}}{1-\lambda f_{\gamma}}=\frac{\sum_{k=0}^{\gamma}\left(\begin{array}{l}\gamma \\ k\end{array}\right)(-1)^{k} f_{k}}{1-\lambda f_{\gamma}}$.

Since $f(z)$ is the probability that the quark $q^{\prime}$ has the fraction of momentum $z$ of the quark $q$ we have $f(z) \geqq 0$ and of course from Eq. (29)

$\int_{0}^{1} d z f(z)=1$,

\footnotetext{
${ }^{3}$ If unambiguous, we use the abbreviation $d(z)=d(z, \rho=0)$, etc.
}

which implies

$f_{\gamma} \gtrless f_{0}=1$ for $\gamma \lessgtr 0$.

The first two moments are then

$d_{0}^{\lambda}=\frac{1}{1-\lambda} ; d_{1}^{\lambda}=\frac{1-f_{1}}{1-\lambda f_{1}}$.

For power behaved primordial functions

$f(z)=\alpha z^{\alpha-1}$

we have

$f_{\gamma}=\frac{\alpha}{\alpha+\gamma} ; f_{\gamma}^{-}=\frac{\Gamma(\alpha+1) \Gamma(\gamma+1)}{\Gamma(\alpha+\gamma+1)}$,

$g_{\gamma}^{\lambda}=\frac{\alpha}{\alpha(1-\lambda)+\gamma}$,

$d_{\gamma}^{\lambda}=\frac{\Gamma(\alpha+1) \Gamma(\gamma+1)}{\Gamma(\alpha+\gamma)} \frac{1}{\alpha(1-\lambda)+\gamma}$.

The complete solution in the power case is, as one can easily check

$g^{\lambda}(z)=\alpha z^{(1-\lambda) \alpha-1}$

and so

$g^{(n)}(z)=\frac{\alpha^{n}}{(n-1) !}(-\ln z)^{n-1} z^{\alpha-1}$.

The meson distribution is then found by inserting $g^{\lambda}(z)$ into Eq. (39)

$d^{\lambda}(z) \underset{\alpha>1}{=} \alpha(\alpha-1) \int_{z}^{1} d \xi(\xi-z)^{\alpha-2} \xi^{-\alpha \lambda}$,

$d^{\lambda}(z) \underset{\alpha=1}{=} z^{-\lambda}$.

For $\lambda=1$ this gives the well-known result

$d^{1}(z)=\frac{\alpha}{z}(1-z)^{\alpha-1}$.

We have also determined the solutions for the more general case of a primordial function consisting of a sum of two powers. The results are given in the appendix.

Sukhatme [6] emphasized the importance of the behaviour of $d^{(n)}(z)$ for $z \rightarrow 1$ because this determines near $z=1$ the ratio of fragmentation functions for mesons, which can be produced in the first breakup and those which cannot (ratio of favoured to unfavoured decays). One can prove that

$g^{(n)}(z) \underset{z \rightarrow 1}{\longrightarrow} \frac{f(1)^{n}}{(n-1) !}(1-z)^{n-1}$,

$d^{(n)}(z) \underset{z \rightarrow 1}{\longrightarrow} \frac{\Gamma(\delta)}{\Gamma(\delta+n-1)} f(1)^{n-1}(1-z)^{n-1} f(1-z)$,

where $\delta$ is taken from the behaviour of $f(z)$ near $z=0$

$f(z) \underset{z \rightarrow 0}{\sim} z^{\delta-1} \ln ^{a} z$, 
which any physically reasonable primordial function will fulfill. So indeed, in cascade models, one will always have an additional factor $(1-z)$ for the distributions of mesons coming from successive vertices.

\subsection{Primordial Functions of the Type $\alpha z^{\alpha-1} \cdot T\left(\mathbf{p}_{T}\right)$}

There is one case, fortunately a useful one, where we can solve Eqs. (34-37) exactly, that is when the primordial function is of the type

$F\left(z ; \mathbf{p}_{T}\right)=\alpha z^{\alpha-1} T\left(\mathbf{p}_{T}\right)$,

with, by Eq. (29)

$\int d^{2} p_{T} T\left(\mathbf{p}_{T}\right)=1$.

The ansatz Eq. (60) is the straightforward three dimensional generalization of the one dimensional case Eq. (50). Denoting as before the Fourier transform

$t(\boldsymbol{\rho})=\int d^{2} p_{T} e^{i \mathbf{p}_{T} \cdot \boldsymbol{\rho}} T\left(\mathbf{p}_{T}\right) ; t(0)=1$,

one obtains from Eq. (34)

$g^{\lambda}(z, \rho)=\alpha z^{\alpha-1} t(\rho) \exp \left[\alpha \lambda \int_{z}^{1} \frac{d \xi}{\xi} t(\rho \xi)\right]$.

Inserting this into Eq. (36) gives

$$
\begin{aligned}
& d^{\lambda}(z, \rho) \\
& =\alpha t(-\rho)\left\{(1-z)^{\alpha-1}+\alpha \lambda \int_{z}^{1} \frac{d \xi}{\xi}(\xi-z)^{\alpha-1} t\left(\frac{z}{\xi} \rho\right)\right. \\
& \left.\cdot \exp \left[\alpha \lambda \int_{\xi}^{1} \frac{d \eta}{\eta} t\left(\frac{z}{\eta} \rho\right)\right]\right\}, \\
& d^{\lambda}(z, \rho)=\left\{\begin{array}{l}
\alpha(\alpha-1) t(-\rho) \int_{z}^{1} d \xi(\xi-z)^{\alpha-2} \\
\exp \left[\alpha \lambda \int_{\xi}^{1} \frac{d \eta}{\eta} t\left(\frac{z}{\eta} \rho\right)\right] \text { if } \alpha>1 \\
t(-\rho) \exp \left[\lambda \int_{z}^{1} \frac{d \xi}{\xi} t(\xi \rho)\right] \text { if } \alpha=1 .
\end{array}\right.
\end{aligned}
$$

Observe, that for $\rho=0$, we obtain the one dimensional solution, Eq. (56), as we should.

To obtain $D^{\lambda}\left(z ; \mathbf{p}_{T}\right)$ we transform back; $\alpha>1$, $t(\boldsymbol{\rho})=t(-\boldsymbol{\rho})=t(\rho)$ assumed, leads to

$$
D^{\lambda}\left(z ; \mathbf{p}_{T}\right)=\frac{1}{2 \pi} \int_{0}^{\infty} d \rho \rho J_{0}\left(p_{T} \rho\right) d^{\lambda}(z, \rho)
$$

\subsection{Factorizing Primordial Functions and the Seagull Effect}

Consider the more general case of a factorizing primordial function

$F\left(z ; \mathbf{p}_{T}\right)=f(z) T\left(\mathbf{p}_{T}\right)$ general case of physical interest, we would like at least to have a good approximation of $D^{\lambda}\left(z ; \mathbf{p}_{T}\right)$ in this case. To this end we insert $F$ from Eq. (67) in Eq. (34) and (37) and eliminate $f(z)$ from these equations, resulting into

$$
\begin{aligned}
& d^{\lambda}(z, \rho)=t(-\boldsymbol{\rho}) d^{\lambda}(z, 0) \\
& +\lambda \int_{z}^{1} \frac{d \xi}{\xi}[t(\xi \rho)-1] d^{\lambda}(z, \rho) g^{\lambda}\left(\frac{z}{\xi}, 0\right),
\end{aligned}
$$

which for small $\rho$ evidently reads (as $t(0)=1$ ):

$$
\begin{aligned}
& d^{\lambda}(z, \rho) \underset{\rho \rightarrow 0}{=} t(-\rho) d^{\lambda}(z, 0) \\
& +\lambda \int_{z}^{1} \frac{d \xi}{\xi}[t(\xi \rho)-1] d^{\lambda}(z, 0) g^{\lambda}\left(\frac{z}{\xi}, 0\right) .
\end{aligned}
$$

So we see, that for small $\rho$ we can express $d^{\lambda}(z, \boldsymbol{\rho})$ in terms of the solution at $\rho=0$, which is given by the one dimensional equations.

Being only interested in functions $T\left(\mathbf{p}_{T}\right)$ with the properties

$T\left(\mathbf{p}_{T}\right)=T\left(p_{T}\right) ; 0<\int d^{2} p_{T} p_{T}^{2} T\left(p_{T}\right) \equiv \overline{p_{T}^{2}}<\infty$

we can assume that

$t(\rho)=t(\rho) \underset{\rho \rightarrow 0}{=} 1-\rho^{2} \overline{p_{T}^{2}} / 4$.

Inserting the last equation into Eq. (69) we obtain for the ratio of the variances in $p_{T}$ of $D^{\lambda}\left(z ; \mathbf{p}_{T}\right)$ and $F\left(z ; \mathbf{p}_{T}\right)$

$r^{\lambda}(z) \equiv \frac{\left\langle p_{T}^{2}\right\rangle^{\lambda}}{\overline{p_{T}^{2}}}=1+\lambda \frac{\int_{z}^{1} d \xi \xi d^{\lambda}(\xi) g^{\lambda}\left(\frac{z}{\xi}\right)}{d^{\lambda}(z)} ;$

i.e. we observe the seagull effect $(z$ dependence of $\left.\left\langle p_{T}^{2}\right\rangle^{\lambda}\right)$ and its independence of the form of $T\left(\mathbf{p}_{T}\right)$. This $z$ dependence can be calculated exactly from Eq. (72) by solving the one dimensional equations. One can easily check that

$r^{\lambda}(1)=1$,

and

$r^{\lambda 1}(1)=-\lambda f(1) / \delta$,

where $\delta$ is defined by Eq. (59).

As an example, we give the result for primordial functions of the type (60)

$r^{\lambda}(z)=1+\frac{\alpha \lambda}{2} \frac{\int_{z}^{1} d x(1-x)^{\alpha-2} x^{\alpha(\lambda-1)}\left(x^{2}-z^{2}\right)}{\int_{z}^{1} d x(1-x)^{\alpha-2} x^{\alpha(\lambda-1)}} ; \alpha \geqq 1$, 
which for integer $\alpha$ leads to

$$
\left\{\begin{array}{l}
r^{\lambda}(z)=\left[\begin{array}{c}
\sum_{K=0}^{\alpha-2}\left(\begin{array}{c}
\alpha-2 \\
K
\end{array}\right) \frac{(-1)^{K}}{\alpha(\lambda-1)+K+3} \\
1+\left[\begin{array}{c}
\alpha \lambda \cdot\left(1-z^{\alpha(\lambda-1)+K+3}\right) \\
2 \sum_{K=0}^{\alpha-2}\left(\begin{array}{c}
\alpha-2 \\
K
\end{array}\right) \frac{(-1)^{K}}{\alpha(\lambda-1)+K+1} \\
\cdot\left(1-z^{\alpha(\lambda-1)+K+1}\right)
\end{array}\right. \\
\text { for } \alpha=2,3 \ldots \\
1+\frac{\lambda}{2}\left(1-z^{2}\right) ; \text { for } \alpha=1
\end{array}\right] ;
\end{array}\right.
$$

with the understanding $\frac{1-z^{\varepsilon}}{\varepsilon} \underset{\varepsilon \rightarrow 0}{\longrightarrow}-\ln z$. For $\lambda<0$ $(>0) r^{\lambda}(z)$ is a concave (convex) curve in $z$. To obtain a good approximation for $D^{\lambda}\left(z ; \mathbf{p}_{T}\right)$ in the more general case of a factorizing primordial function we now simply change the width of the transverse part $T\left(\mathbf{p}_{T}\right)$ to the required width given by Eq. (72)

$D^{\lambda}\left(z ; \mathbf{p}_{T}\right) \approx d^{\lambda}(z) \frac{T\left(\frac{\mathbf{p}_{T}}{\sqrt{r^{\lambda}(z)}}\right)}{r^{\lambda}(z)}$.

In doing this we are sure that $D^{\lambda}\left(z ; \mathbf{p}_{T}\right)$ has the right width in $p_{T}$ and is exact when integrated over $\mathbf{p}_{T}$. We shall test this approximation for two examples of $T\left(\mathbf{p}_{T}\right)$ in the next section.

\section{Numerical Examples}

The most commonly used transverse momentum dependences are gaussian and exponential

$$
\begin{aligned}
& T_{G}\left(\mathbf{p}_{T}\right)=\frac{\exp \left(-p_{T}^{2} / \overline{p_{T}^{2}}\right)}{\pi \overline{p_{T}^{2}}}, \\
& T_{E}\left(\mathbf{p}_{T}\right)=3 \frac{\exp \left(-p_{T} \sqrt{6 / \overline{p_{T}^{2}}}\right)}{\pi \overline{p_{T}^{2}}},
\end{aligned}
$$

implying

$t_{G}(\rho)=\exp \left(-\rho^{2} \overline{p_{T}^{2}} / 4\right)$

$t_{E}(\boldsymbol{\rho})=\left(1+\boldsymbol{\rho}^{2} \overline{p_{T}^{2}} / 6\right)^{-3 / 2}$.

Following Field and Feynman [5] we have chosen

$f(z)=3 z^{2}$,

i.e. $\alpha=3$, for the $z$ dependence of the primordial function. Equations (65) and (66) have then been evaluated for several $\lambda(\lambda=1,-1 / 3,2 / 5)$ and $p_{T}^{2}=0.25$. In Figure 2(3) we show the comparison of these calculations with the results of the approximation formula, Eq. (77), for $\lambda=1 ; z=0.25,0.5,0.75$ and gaussian (exponential) $T$-functions. The $\lambda$-values $-1 / 3$ and $2 / 5$ lead to curves, which cannot be distinguished from their corresponding approximations.

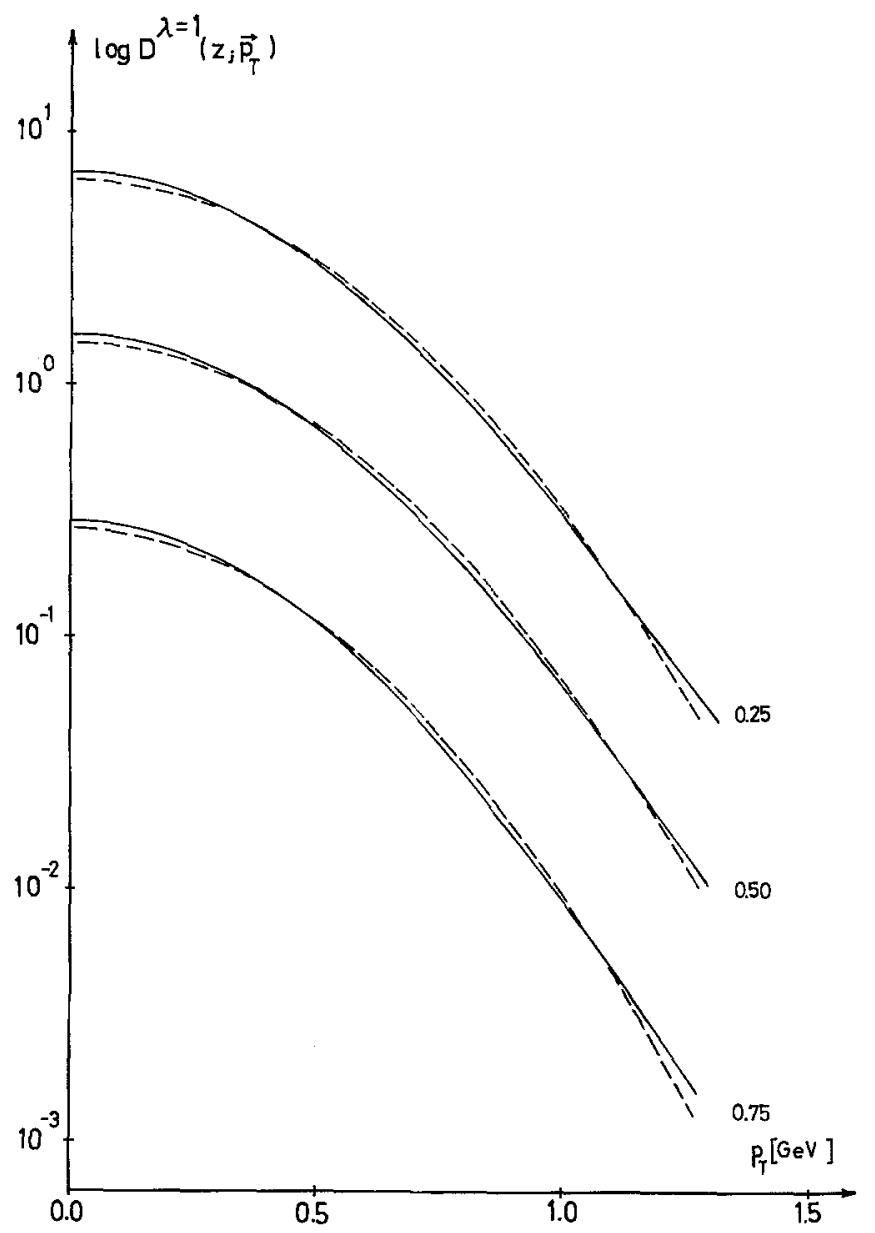

Fig. 2. Logarithm of $D^{\lambda=1}\left(z ; \mathbf{p}_{T}\right)$ as a function of $p_{T} ;$ the primordial function was $3 z^{2} \cdot T_{G}\left(\mathbf{p}_{T}\right)$. The solid lines are the exact results, the broken lines the approximation (77), the numbers indicate the $z$-values, $\overline{p_{T}^{2}}=0.25$

In Figure 4 we present the seagull functions for these values of $\lambda$, as taken from Eq. (76). At first sight the effect does not seem to be very important, however, the combination of different $\lambda$-values in special models can enhance the effect considerably. To show this, we have considered the models proposed by Niedermayer [3] and Sukhatme [6].

Niedermayer takes only the $u$ and $d$ flavours; his probability matrix is

$P_{N}=\left(\begin{array}{ll}1 / 3 & 2 / 3 \\ 2 / 3 & 1 / 3\end{array}\right)$

with eigenvalues $\lambda_{1}=1, \lambda_{2}=-1 / 3$, from which we calculate [using Eq. (23)]

$D_{u}^{\pi \pm}=\frac{1}{3}\left(D^{\lambda=1} \pm D^{\lambda=-1 / 3}\right)$.

In Sukhatme's model the $s$ flavour is also included; the probability matrix is

$P_{S}=\frac{1}{5}\left(\begin{array}{lll}2 & 2 & 1 \\ 2 & 2 & 1 \\ 1 & 1 & 3\end{array}\right)$ 


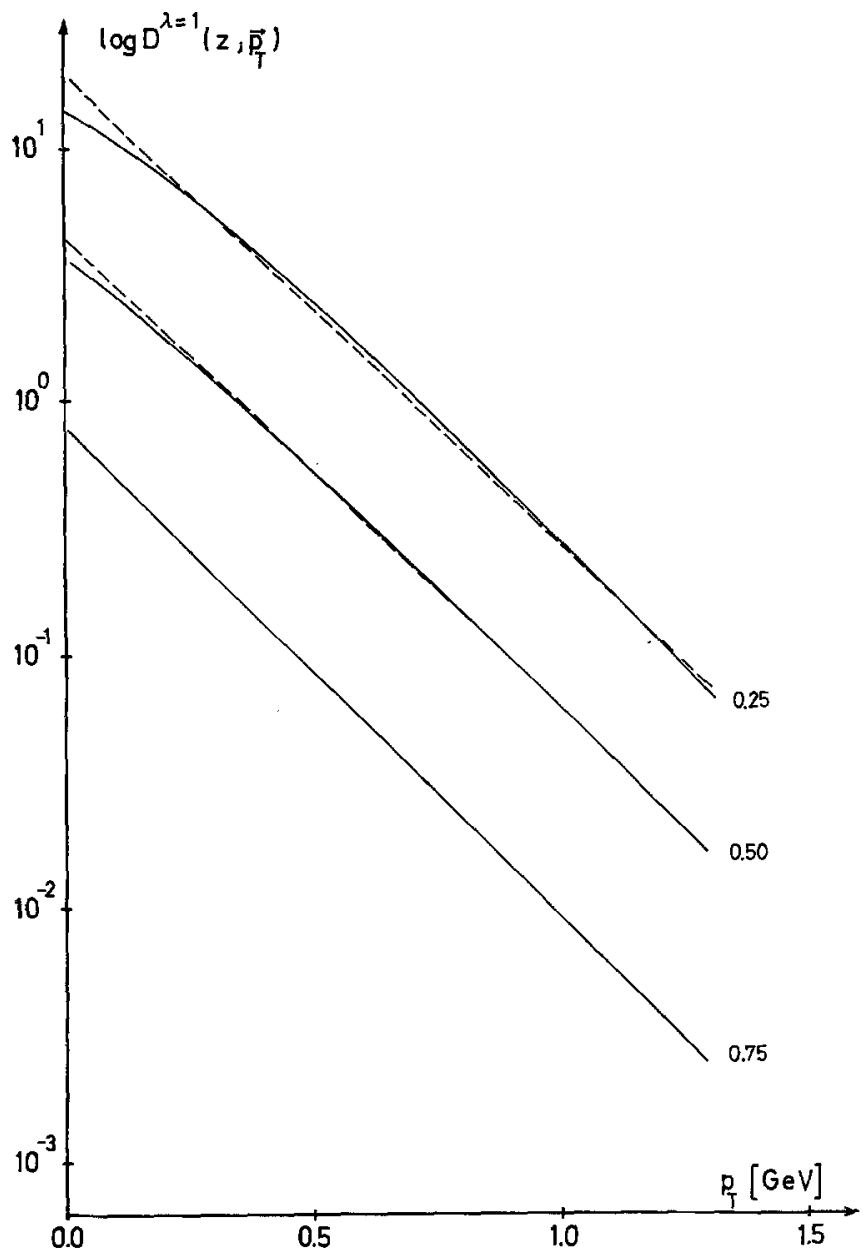

Fig. 3. Logarithm of $D^{\lambda=1}\left(z ; \mathbf{p}_{T}\right)$ as a function of $p_{T}$; the primordial function was $3 z^{2} \cdot T_{E}\left(\mathbf{p}_{T}\right)$. The notation is the same as in Figure 2 and has the eigenvalues $\lambda_{1}=1, \lambda_{2}=0, \lambda_{3}=2 / 5$. Taking only the directly produced $\pi^{ \pm}$into account, i.e. no $\eta, \eta^{\prime}$ decay, we find

$D_{u}^{\pi \pm}=\frac{1}{15}\left(2 D^{\lambda=1} \pm 3 D^{\lambda=0}+D^{\lambda=2 / 5}\right)$.

The average $\mathbf{p}_{T}^{2}$ belonging to any combination of $D^{\lambda_{i}}$

$D=\sum_{i} \beta_{i} D^{\lambda_{i}}$

is given by

$\left\langle p_{T}^{2}\right\rangle=\overline{p_{T}^{2}} \frac{\sum_{i} \beta_{i} r^{\lambda_{i}}(z) d^{\lambda_{i}}(z)}{\sum_{i} \beta_{i} d^{\lambda_{i}}(z)}$,

thus the total $\left\langle p_{\tau}^{2}\right\rangle$ is the averaged $\left\langle p_{T}^{2}\right\rangle^{\lambda_{i}}$ with weights $\beta_{i} d^{\lambda_{i}}(z)$. From formula (88) and the weights given according to Eqs. (84) and (86) we calculated $r_{u}^{\pi \pm}(z)$ [as before with the ansatz (82)]. The result is shown in Figure 5. We observe, that the two models show a similar behaviour. In both cases the production of the unfavoured meson $\left(\pi^{-}\right)$exhibits a strong seagull effect. At $z=1$ the ratio $r_{u}^{\pi^{-}}$even reaches the value 2. In fact, it can be shown (Appendix), that for factorizing primordial functions all unfavoured decays will lead to

$r_{q}^{\text {unfavoured }}(z=1)=2$.

Thus the seagull effect will be an important phenomenon in all these models.

We are indebted to R. Baier and $\mathbf{J}$. Cleymans for helpful discussions.

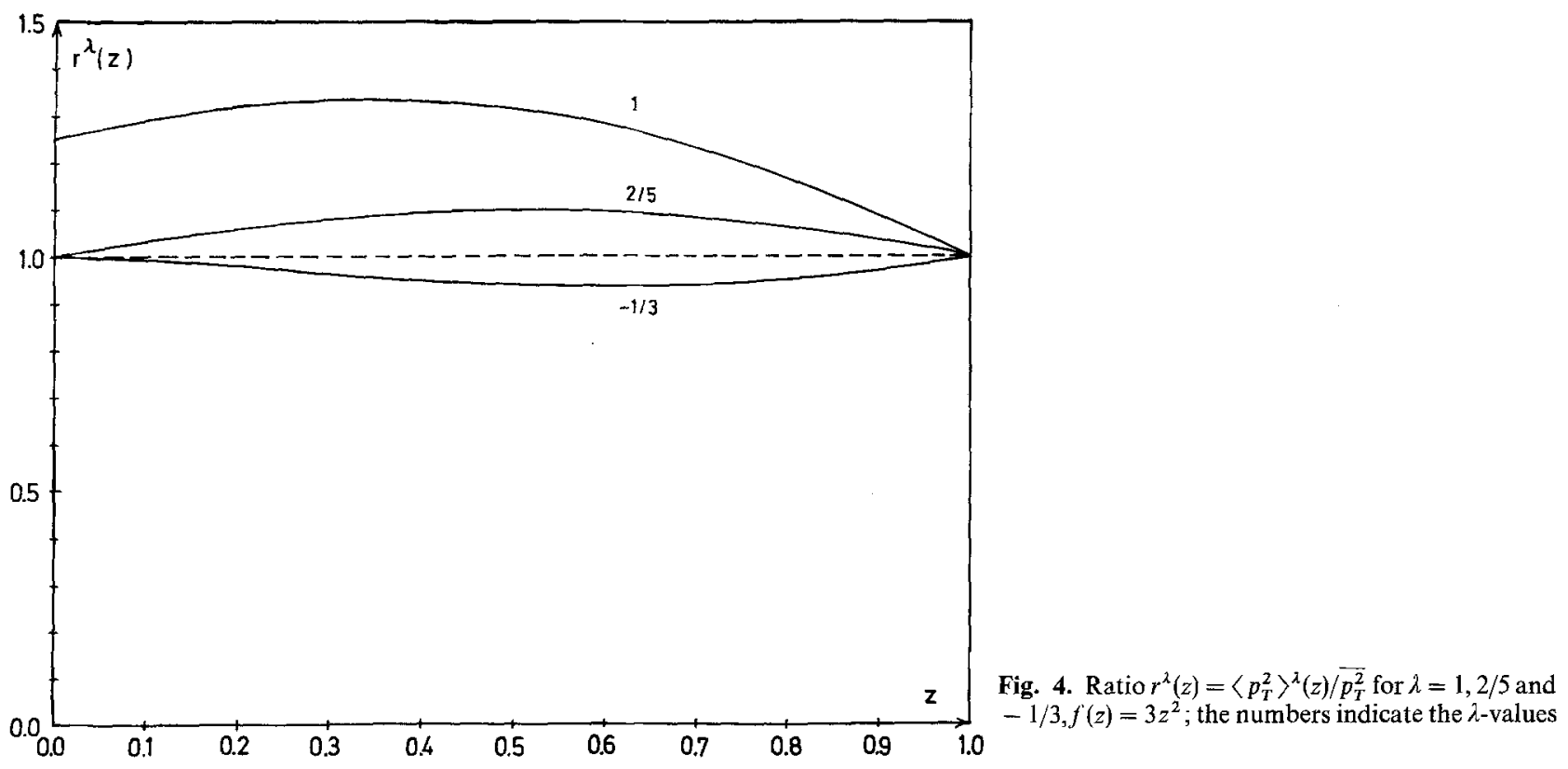




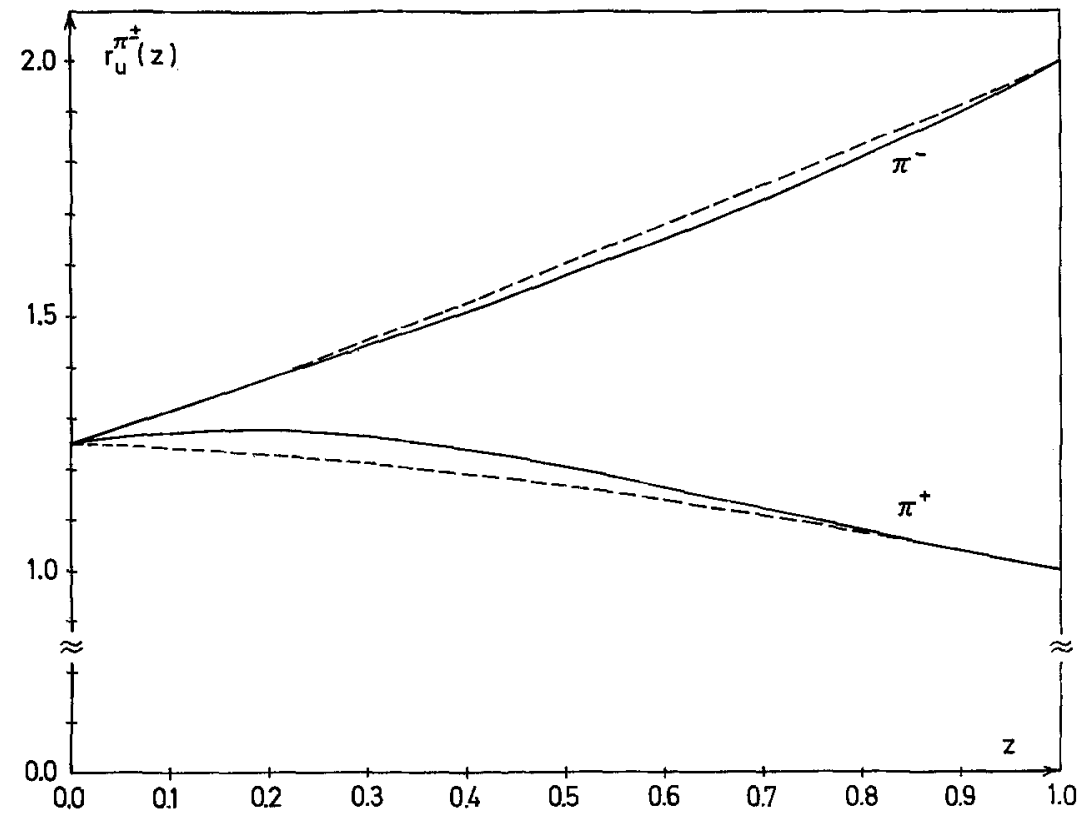

Fig. 5. Ratio $r_{u}^{\pi \pm}(z)$ calculated for the models [3] (solid lines) and [6] (broken lines)

\section{Summary and Conclusions}

We presented a covariant generalization of the one dimensional cascade model for quark fragmentation into a jet of mesons. By introducing generating functions we were able to treat any number of quark flavours. In the scaling limit, the full solution for primordial functions of the type $\alpha z^{\alpha-1} T\left(p_{T}\right)$ was found. For all factorizing primordial functions it was shown that there is a seagull effect and we derived an expression, from which it can be calculated, once the one dimensional solution is known. It turned out, that the seagull is independent of the form of the transverse momentum part of the factorizing primordial function. At $z=1$ the ratio $\left\langle p_{T}^{2}\right\rangle / \bar{p}_{T}^{2}$ is 2 for unfavoured and 1 for favoured decays of any quark, independent of the probability matrix used. For the soluable model we demonstrated how well our approximation for $D^{\lambda}\left(z ; \mathbf{p}_{T}\right)$ works and we believe the approximation will do as well for more complicated longitudinal factors in the primordial function. Therefore, we propose the following recipe to include transverse momentum into one dimensional models:

i) determine the eigenvalues and eigenvectors of your probability matrix;

ii) solve the one dimensional equation (38) for $g^{\lambda}(z)$; iii) calculate $d^{\lambda}(z), r^{\lambda}(z)$ from (39) and (72);

iv) take the approximation (77) and compose all quark functions from Eq. (23);

v) if you have decaying mesons, fold their $D$ 's with the decay distribution and add the result to the corresponding $D$ 's from direct production.

\section{Appendix}

\section{A. The Two Power Case}

$f(z)=\alpha_{1} \beta_{1} z^{\alpha_{1}-1}+\alpha_{2} \beta_{2} z^{\alpha_{2}-1}, \quad$ with $\beta_{1}+\beta_{2}=1$.

By applying the method described in the text one readily finds

$g^{\lambda}(z)=A^{+} z^{V+-1}+A^{-} z^{V--1}$,

where $V_{ \pm}$are the roots of

$V^{2}-V\left[\alpha_{1}+\alpha_{2}-\lambda\left(\alpha_{1} \beta_{1}+\alpha_{2} \beta_{2}\right)\right]+(1-\lambda) \alpha_{1} \alpha_{2}=0$

and

$A^{ \pm}=\frac{-\alpha_{1} \alpha_{2}+\left(\alpha_{1} \beta_{1}+\alpha_{2} \beta_{2}\right) V_{ \pm}}{V^{ \pm}-V^{\bar{\mp}}}$.

To obtain $d^{\lambda}(z)$ we now insert $g^{\lambda}(z)$ into Eq. (39) and partially integrate (only valid if $\alpha_{i}>1$ ), giving

$$
\begin{aligned}
& d^{\lambda}(z)=\frac{\lambda A^{+} \alpha_{1} \beta_{1}}{\alpha_{1}-V_{+}}\left(\alpha_{1}-1\right) \int_{z}^{1} d \xi(\xi-z)^{\alpha_{1}-2} \xi^{V+-\alpha_{1}} \\
& +\frac{\lambda A^{+} \alpha_{2} \beta_{2}}{\alpha_{2}-V_{+}}\left(\alpha_{2}-1\right) \int_{z}^{1} d \xi(\xi-z)^{\alpha_{2}-2} \xi^{V+-\alpha_{2}} \\
& +\frac{\lambda A^{-} \alpha_{1} \beta_{1}}{\alpha_{1}-V_{-}}\left(\alpha_{1}-1\right) \int_{z}^{1} d \xi(\xi-z)^{\alpha_{1}-2} \xi^{V--\alpha_{1}} \\
& +\frac{\lambda A^{-} \alpha_{2} \beta_{2}}{\alpha_{2}-V_{-}}\left(\alpha_{2}-1\right) \int_{z}^{1} d \xi(\xi-z)^{\alpha_{2}-2} \xi^{V--\alpha_{2}}
\end{aligned}
$$

This formula can also be used, if one of the $\alpha_{i}^{\text {'s is }}$ 
equal to 1 , as

$\lim _{\alpha \downarrow 1}(\alpha-1) \int_{z}^{1} d \xi(\xi-z)^{\alpha-2} q(\xi)=q(z)$.

For integer $\alpha$ 's the integrals in (A.5) are all elementary.

B. $\left\langle p_{T}^{2}\right\rangle$ for $z=1$ in the Favoured and Unfavoured Case

By Eq. (88)

$\left\langle p_{T}^{2}\right\rangle=\overline{p_{T}^{2}} \frac{\sum_{i} \beta_{i} d^{\lambda_{i}}(z) r^{\lambda_{i}}(z)}{\sum_{i} \beta_{i} d^{\lambda_{i}}(z)}$, where

$\sum_{i} \beta_{i} \neq 0$ in favoured case

$\sum_{i} \beta_{i}=0$ in unfavoured case.

So we need the behaviour of $d^{\lambda_{i}}$ and $r^{\lambda_{i}}$ for $z \uparrow 1$. From Eq. (38) we see that

$g^{\lambda}(1)=f(1)$

and so by Eq. (39)

$d^{\lambda}(z)=f(1-z)+\lambda f(1) \int_{z}^{1} d \xi f(1-\xi)$.

Inserting this into Eq. (72) one obtains $r^{\lambda}(z)$ for $z \uparrow 1$ $r^{\lambda}(z) \underset{z \pi 1}{=1} 1+\lambda f(1) \frac{\int_{z}^{1} d \xi f(1-\xi)}{f(1-z)}=\frac{d^{\lambda}(z)}{f(1-z)}$.

So Eq. (B.1) now reads for $z \uparrow 1$

$$
\left\langle p_{T}^{2}\right\rangle \underset{z \uparrow 1}{=} \overline{p_{T}^{2}} \frac{\sum \beta_{i}\left\{r^{\lambda_{i}}(z)\right\}^{2}}{\sum \beta_{i} r^{\lambda_{i}}(z)} \underset{\text { as } r^{\lambda_{i}(1)=1}=1}{=}\left\{\begin{array}{l}
2 \overline{p_{T}^{2}} \text { if } \sum \beta_{i}=0 \\
\overline{p_{T}^{2}} \text { if } \sum \beta_{i} \neq 0 .
\end{array}\right.
$$

So we see that

$\left\langle p_{T}^{2}\right\rangle(z=1)=\left\{\begin{array}{l}2 \overline{p_{T}^{2}} \text { in the unfavoured case } \\ \overline{p_{T}^{2}} \text { in the favoured case }\end{array}\right.$

\section{References}

1. Hanson, G. : Invited talk at the XIIIth Rencontre de Moriond, Les Arcs, March 1978

2. PLUTO Collaboration: Phys. Lett. 78B, 176 (1978)

3. Niedermayer, F. : Nucl. Phys. B79, 355 (1974)

4. Feynman, R.P. : Proc. VIIIth Intern. Symp. on Multiparticle Dynamics Kaysersberg, 1977

5. Field, R.D., Feynman, R.P. : Nucl. Phys. B136, 1 (1978)

6. Sukhatme, U.P. : Phys. Lett. 73B, 478 (1978);

Sukhatme, U.P.: Talk given at the XIIIth Rencontre de Moriond, Les Arcs, March 1978

7. Feynman, R.P.: Photon-hadron interactions. Reading, MA., Benjamin, W.A., 1972

8. Cahn, R.N., Colglazier, E.W. : Phys. Rev. D9, 2658 (1974)

9. Bjorken, J.D., Farrar, G.R. : Phys. Rev. D9, 1449 (1974)

10. Krzywicki, A., Petersson, B. : Phys. Rev. D6, 924 (1972)

11. Finkelstein, J., Peccei, R.D. : Phys. Rev. D6, 2606 (1972)

Received 18 July 1978

Note Added in Proof. The following comment to Eq. (2) is appropriate.

We take all quarks to be on mass-shell with equal masses. As a consequence the mesons are off-shell, which, however, is no problem in the scaling limit, where masses are neglected. A possible interpretation is that (analogous to Bremsstrahlung) the exchange of a soft quantum with the (confining) field brings the meson on-shell. 